\title{
Low-cost wireless power efficiency optimization of the NFC tag through switchable receiver antenna - ERRATUM
}

An earlier version of the above mentioned article [1] was published with an incorrect expansion of the acronym WISP. This has now been corrected.

REFERENCE

[1] Zhao, Y.; Li, H.; Naderiparizi, S.; Parks, A.; Smith, J.R.: Low-cost wireless power efficiency optimization of the NFC tag through switchable receiver antenna. Wireless Power Transfer (2018), 1-10. doi:10.1017/wpt.2018.1 